\title{
ALDEHYDE DEHYDROGENASE 3A1 PROTECTS AIRWAY EPITHELIAL CELLS FROM CIGARETTE SMOKE-INDUCED DNA DAMAGE AND CYTOTOXICITY
}

\author{
Jun-Ho Jang ${ }^{1,2}$, Shannon Bruse ${ }^{2}$, Yushi Liu², Veronica Duffy ${ }^{2}$, Chunyu Zhang ${ }^{2}$, Nathaniel \\ Oyamada $^{2}$, Scott Randell ${ }^{3}$, Akiko Matsumoto ${ }^{4}$, David C. Thompson ${ }^{4}$, Yong Lin ${ }^{2}$, Vasilis \\ Vasiliou $^{4}$, Yohannes Tesfaigzi ${ }^{2}$, and Toru Nyunoya ${ }^{1,2}$ \\ ${ }^{1}$ Division of Pulmonary, Critical Care Medicine, Department of Internal Medicine, University of \\ New Mexico and New Mexico VA Health Care System \\ ${ }^{2}$ COPD Program, Lovelace Respiratory Research Institute, Albuquerque, NM \\ ${ }^{3}$ Department of Cell and Molecular Physiology, University of North Carolina, Chapel Hill, NC \\ ${ }^{4}$ Department of Pharmacology, University of Denver Colorado, Aurora, CO
}

\section{Abstract}

\begin{abstract}
Aldehyde dehydrogenase 3A1 (ALDH3A1), an ALDH superfamily member, catalyzes the oxidation of reactive aldehydes, highly toxic components of cigarette smoke (CS). Even so, the role of ALDH3A1 in CS-induced cytotoxicity and DNA damage has not been examined. Among all of the ALDH superfamily members, ALDH3A1 mRNA levels showed the greatest induction in response to CS extract (CSE) exposure of primary human bronchial epithelial cells (HBECs). ALDH3A1 protein accumulation was accompanied by increased ALDH enzymatic activity in CSE-exposed immortalized HBECs. The effects of overexpression or suppression of ALDH3A1 on CSE-induced cytotoxicity and DNA damage $(\gamma \mathrm{H} 2 \mathrm{AX})$ were evaluated in cultured immortalized HBECs. Enforced expression of ALDH3A1 attenuated cytotoxicity and downregulated $\gamma \mathrm{H} 2 \mathrm{AX}$. siRNA-mediated suppression of ALDH3A1 blocked ALDH enzymatic activity and augmented cytotoxicity in CSE-exposed cells. Our results suggest that the availability of ALDH3A1 is important for cell survival against CSE in HBECs.
\end{abstract}

\author{
Keywords \\ cigarette smoke; airway epithelial cells; chronic obstructive pulmonary disease; ALDH3A1; \\ reactive aldehydes; DNA damage; cytotoxicity; FANCD2
}

\section{INTRODUCTION}

Cigarette smoke (CS) contains abundant reactive aldehydes [1]. In contrast to reactive oxygen species (ROS), aldehydes are relatively long-lived and highly cell permeable, allowing them to cause distant target effects, including DNA damage, glutathione depletion,

Crown Copyright $\odot 2013$ The Authors. Published by Elsevier Inc. All rights reserved.

Address correspondence to: Toru Nyunoya, M.D., Lovelace Respiratory Research Institute, 2425 Ridgecrest Drive SE, Albuquerque, NM 87108. Tel: (505) 348-9366, Fax: (505) 348-8346, tnyunoya@lrri.org.

Publisher's Disclaimer: This is a PDF file of an unedited manuscript that has been accepted for publication. As a service to our customers we are providing this early version of the manuscript. The manuscript will undergo copyediting, typesetting, and review of the resulting proof before it is published in its final citable form. Please note that during the production process errors may be discovered which could affect the content, and all legal disclaimers that apply to the journal pertain. 
enzyme inactivation and cell death [2]. The aldehyde dehydrogenase (ALDH) superfamily is composed of $\mathrm{NAD}(\mathrm{P})^{+}$-dependent enzymes which catalyze aldehyde oxidation. To date, nineteen ALDH genes have been identified in the human genome [2]. Several studies have demonstrated that human bronchial epithelial cells (HBECs) and lung tissues obtained from smokers exhibit a marked increase in ALDH3A1 gene and protein expression compared to those from nonsmokers [3-6]. However, the biological relevance of cigarette smoke-induced ALDH3A1 upregulation in HBECs has not been examined.

ALDH3A1 is a $54 \mathrm{kDa}$ protein which is constitutively expressed as a homodimer in various tissues, including cornea, lung, esophagus and stomach [2]. ALDH3A1 gene expression can be induced by various xenobiotics, such as polycyclic aromatic hydrocarbons (PAHs) and 2,3,7,8-tetrachlorodibenzo-p-dioxin (TCDD; dioxin), through aryl hydrocarbon receptor (AHR)- or non-AHR-dependent mechanisms [3-5]. ALDH3A1 catalyzes the oxidation of aliphatic, aromatic, and lipid peroxidation (LPO)-derived aldehydes, including 4-hydroxy-2nonenal (4-HNE) [2]. ALDH3A1 also attenuates LPO-mediated growth inhibition [6], UV light-induced cytotoxicity [7], ROS-induced protein modification [8], and genotoxininduced DNA damage and apoptosis [9].

CS exposure induces the DNA damage response (DDR) that is mediated by phosphoinositide 3-kinase related protein kinases (PIKKs) [10-11]. Upon DNA damage, the PIKKs phosphorylate the serine 139 residue of $\mathrm{H} 2 \mathrm{AX}$ variant $(\gamma \mathrm{H} 2 \mathrm{AX})$ on chromatin flanking DNA double-strand break (DSB) sites. This phosphorylation has been widely used as a sensitive marker of DNA damage [12-13]. $\gamma \mathrm{H} 2 \mathrm{AX}$ is crucial for the subsequent DDR signaling and DNA repair [14]. The DDR signaling and activation of PIKKs also inhibits cyclin-dependent kinase (CDK) activity by activating p53 and protein kinases, such as CHK1 and CHK2. Suppression of CDK activity delays cell cycle progression and allows the cells to repair DNA damage [10,14-16].

Reactive aldehydes, such as 4-HNE, induce DNA interstrand crosslinks (ICLs) [17] that are detrimental to the cell because they block both transcription and replication through inhibition of DNA double-strand unwinding [18]. The Fanconi anemia (FA) pathway, consisting of 14 complementation groups, plays an important role in ICL repair [18]. FANCD2, a member of the FA complementation group (FANC) counteracts aldehydeinduced DNA damage and cytotoxicity both in vitro and in vivo [19]. Exposure of immortalized HBECs to CS extract (CSE) downregulates FANCD2 protein expression, a change that is detrimental as FANCD2 is required to protect against CSE-induced cytotoxicity [20].

In the present study, we show that among all of the ALDH isozymes, ALDH3A1 exhibits the greatest induction in response to CSE exposure in primary HBECs, and that this induction is mediated by AHR. CSE-exposed immortalized HBECs exhibit a marked increase in ALDH enzymatic activity. ALDH3A1 overexpression attenuates CSE-induced cytotoxicity and DNA damage. Suppression of ALDH3A1 both obstructs ALDH enzymatic activity and augments cytotoxicity induced by CSE. These data suggest that ALDH3A1 modulates CS-induced cytotoxicity and DNA damage in HBECs.

\section{METHODS Cell Culture}

Primary HBECs were isolated from five nonsmokers and maintained under a protocol approved by the LRRI Institutional Review Board as previously described [21]. HBEC2 cells (immortalized HBECs) were originally generated by Ramirez, et al. [22] and maintained as previously described [23]. Experiments were performed in twelve-well Costar 
tissue culture plates or p100 dishes $(100 \mathrm{~mm})$ at a starting cell density of $10 \times 10^{3} / \mathrm{cm}^{2}$. Cell counts were performed by an electric particle counter (Beckman Coulter, Indianapolis, IN). Twenty-four $\mathrm{h}$ after plating, cells were exposed to various concentrations of CSE for 24 and/ or $48 \mathrm{~h}$.

\section{Cell Viability}

Cell viability was determined by measuring the reduction of 3-(4,5-dimethythiazol-2-

yl)-2,5-diphenyl tetrazolium bromide (MTT) or the trypan blue assay as previously described [24-25]. MTT absorbance was read at $570 \mathrm{~nm}$. CSE-unexposed cells (0\% CSE) with or without siRNA transfection or vector transduction were regarded as $100 \%$ viability. The relative cell viability of CSE-exposed cells was determined by the comparison with CSE-unexposed cells with the same treatment (e.g., scrambled control or ALDH3A1 SiRNA).

\section{Reagents and Antibodies}

Chemicals were obtained from Sigma Chemical (St. Louis, MO) and Calbiochem (La Jolla, CA). Protease inhibitors were obtained from Boehringer Mannheim (St. Louis, MO). Polyvinylidene difluoride membranes were obtained from Bio-Rad (Hercules, CA). ECL Plus was obtained from Amersham (Arlington Heights, IL). Antibodies were obtained from various sources: Anti-ALDH3A1, and anti-AHR primary antibodies were from Santa Cruz Biotechnology (Santa Cruz, CA); anti-FANCD2 primary antibodies were from Epitomics (Burlingame, CA); phosphorylation-specific antibody for H2AX were from Cell Signaling (Beverly, MA); anti- $\beta$ actin was from Sigma Chemical (St. Louis, MO). Secondary antibodies (horseradish peroxidase-conjugated anti-rabbit or anti-mouse Ig) were obtained from Santa Cruz Biotechnology. Tissue culture plates were obtained from Corning (Corning, NY).

\section{Preparation of Cigarette Smoke Extract (CSE)}

100-mm research cigarettes (3R4F) were purchased from the University of Kentucky. CSE solutions were prepared as previously described [26].

\section{Immunoblotting}

Immunoblot analysis was performed as previously described [26]. Equivalent loading was verified by stripping the blot and reprobing with antibodies to $\beta$-actin. In Figures 3, 4, and 5, relative protein expression was quantified by densitometry and normalized to the corresponding input control ( $\beta$-actin) bands. Either empty or scrambled control in the absence of CSE was set to value of 1.0.

\section{Real-time RT-PCR for 19 ALDH Isozymes}

A customized PCR-Array kit measuring the 19 known ALDH isozymes and Actb, was purchased from Qiagen Inc (Valencia, VA). Quantitative analysis of the PCR data was performed according to the manufacturer's instructions. The PCR data were expressed as relative fold difference compared with Actb between CSE-treated and non-treated control cells.

\section{ALDH enzymatic activity assay}

This assay monitors the production of $\mathrm{NAD}(\mathrm{P}) \mathrm{H}$ from $\mathrm{NAD}(\mathrm{P})^{+}$as aldehydes are oxidized by ALDH. Total ALDH activity was measured with some modifications to the method previously described [27]. Harvested cell pellet samples were homogenized in a binding buffer of pH 7.5 (1 M Potassium phosphate monobasic, 1M Potassium phosphate dibasic, 
0.5 M EDTA, 14.26 mM $\beta$-mercaptoethanol) on ice. Homogenates were sonicated (two cycles of 20-second bursts) and incubate for $20 \mathrm{~min}$ on ice, and centrifuged at 100,000 $\mathrm{g}$ for $40 \mathrm{~min}$. The enzymatic reaction in the protein supernatants was initiated and monitored over 5 min by the addition of aldehyde substrate (benzaldehyde) into the reaction buffer containing $0.1 \mathrm{M}$ sodium pyrophosphate, $20 \mathrm{mM} \mathrm{NAD}(\mathrm{P})^{+}, 20 \mathrm{mM}$ pyrazole, $10 \% \beta$ mercaptoethanol, and $50 \mu \mathrm{g}$ of total protein and measured spectrophotometrically by monitoring the production of $\mathrm{NAD}(\mathrm{P}) \mathrm{H}(340 \mathrm{~nm})$. The ALDH activity was expressed as $\mathrm{nmol}$ of $\mathrm{NAD}(\mathrm{P}) \mathrm{H} / \mathrm{mg}$ protein/min.

\section{Establishment of cell lines with ALDH3A1 overexpression}

HBEC2 cells were infected with lentiviral vectors (pReceiver, GeneCopoeia, Rockville, $\mathrm{MD)}$ to overexpress ALDH3A1 protein according to the manufacturer's instructions. The cells were selected with $25 \mu \mathrm{g} / \mathrm{mL}$ hygromycin for $14 \mathrm{~d}$ and all surviving colonies were collected as a pool.

\section{RNA interference}

Small interfering RNA (siRNA) for AHR, ALDH3A1, and the corresponding scrambled (control) siRNA were purchased from Applied Biosystems (Carlsbad, CA). Transfection of siRNA was performed using INTERFERin (Polyplus-tranfection Inc, New York, NY) according to the manufacturer's instruction. The targeted sequences were as follows: AHR siRNA (catalog \#:1199), 5'-GCAUGAUAGUUUUCCGGCUTT-3'; ALDH3A1 siRNA (catalog \#:s1244), 5'-GCAACGACAAGGUGAUUAATT-3'.

\section{Statistical analysis}

The comparison of CSE induction of the 19 known ALDH isozymes was based on the log transformation of the difference between CSE-exposed and control cells. One sample Student's unpaired t-tests were performed. For all comparisons involving multiple treatment groups, one-way ANOVA was used to identify treatment effects. $p$-values, based on calculated contrasts, were used to assess the individual treatment effects. The results are expressed graphically as the mean \pm SEM.

\section{RESULTS}

\section{Primary human bronchial epithelial cells exposed to cigarette smoke extract exhibit a robust increase in ALDH3A1}

Primary HBECs isolated from 5 nonsmoking donors were cultured in the presence or absence of 1.5\% CSE for $24 \mathrm{~h}$. From among 19 ALDH isozymes, exposure to 1.5\% CSE significantly increased expression of ALDH3A1 (103.7-fold), ALDH1A3 (7.8-fold), ALDH3A2 (5.6-fold), ALDH5A1 (3.7-fold), ALDH3B1 (2.5-fold), ALDH2 (2.4-fold), ALDH16A1 (2.0-fold), ALDH9A1 (1.9-fold), and ALDH18A1 (1.9-fold) (Figure 1A). None of the ALDH family isozymes were significantly reduced. ALDH3A1 protein levels were increased at $24 \mathrm{~h}$ in cultured primary HBECs isolated from three donors (Figure 1B); however, this increase was not observed in HFL1 (normal human fetal lung fibroblasts) or primary lung fibroblasts (data not shown), suggesting that the CSE-induced increase in ALDH3A1 is specific to HBECs but not to fibroblasts.

\section{Cigarette smoke extract-induced ALDH3A1 protein accumulation is accompanied by increased ALDH activity}

We next evaluated the effects of CSE on cytotoxicity and ALDH3A1 protein expression in HBEC2 cells. CSE was cytotoxic to HBEC2 cells in a dose- and time-dependent manner (Figure 2A). Similar to the effects of CSE on ALDH3A1 in primary HBECs, CSE increased 
ALDH3A1 protein levels (Figure 2B) but this increase did not occur until $8 \mathrm{~h}$ after exposure (data not shown). The effects of $1.5 \%$ CSE on ALDH enzymatic activity was determined using benzaldehyde, a substrate metabolized by ALDH3A1. Exposure to CSE significantly increased ALDH activity 9.6-fold. While the ALDH activity could have arisen from the increased expression of other ALDH family members, based upon the extent of expression changes, the main contributor for this induction is most likely to be ALDH3A1.

\section{Cigarette smoke extract induces ALDH3A1 via aryl hydrocarbon receptor}

ALDH3A1 is a transcriptional target of AHR [28], Pax6, and Oct1 [5]. To determine if CSE could induce ALDH3A1 through AHR alone, HBEC2 cells were transfected with either siRNA targeting AHR or a scrambled control. Suppression of AHR expression using siRNA (Figure 3A) reduced CSE-induced ALDH3A1 expression by 78\% (Figure 3B). These data suggest that AHR mediates upregulation of ALDH3A1 in response to CSE exposure.

\section{ALDH3A1 attenuates cigarette smoke extract-induced cytotoxicity, DNA damage, and FANCD2 downregulation}

To examine the biological relevance of cigarette smoke-induced ALDH3A1 upregulation, we overexpressed ALDH3A1 in HBEC2 cells using a lentiviral vector and exposed them to various concentrations of CSE for $48 \mathrm{~h}$. When compared to empty vector-exposed cells, ALDH3A1-overexpressing cells were protected from CSE-induced cytotoxicity (Figure 4A) and exhibited reduced DNA damage (as reflected in decreased phosphorylation of H2AX) (Figure 4B).

FANCD2 has been recognized to protect against aldehyde-derived DNA damage [19] and CSE-induced cytotoxicity [20]. Given that FANCD2 is downregulated by CSE [20], we sought to determine whether ALDH3A1 overexpression may influence CSE-induced downregulation of FANCD2 [20]. In these experiments, CSE treatment failed to downregulate FANCD2 expression in ALDH3A1-overexpressing cells (Figure 4C). These data suggest that enforced expression of ALDH3A1 attenuates CS-induced cytotoxicity and DNA damage and that CSE-induced FANCD2 downregulation may be mediated by aldehyde metabolism.

To determine if prior endogenous induction of ALDH3A1 by CSE increased resistance to CSE-induced cytotoxicity, HBEC2 cells were pre-exposed to $0.5 \%$ CSE before the cytotoxic effects of higher concentrations were established (Figure 2A). This CSE concentration was selected because it induces ALDH3A1 (Figure 2B). In these studies, prior induction of ALDH3A1 significantly protected against CSE-induced cytotoxicity (Figure 4D). These data suggest that the availability of ALDH3A1 prior to CSE exposure may be an important determinant of cell protection against CSE.

\section{Suppression of ALDH3A1 levels both blocks ALDH enzymatic activity and augments cytotoxicity induced by cigarette smoke extract}

To further delineate the role of ALDH3A1 in protecting cells from CSE-induced damage, we examined the cytotoxicity of CSE in HBEC2 cells in which ALDH3A1 expression had been suppressed using siRNA. ALDH3A1 siRNA alone significantly reduced cell viability in the absence of CSE (by $59.7 \pm 5.0 \%, p<0.01$ ). Suppression of ALDH3A1 also significantly increased CSE-induced cytotoxicity (Figure 5A). The CSE effects on cell viability determined by the trypan blue assay were similar to those obtained using the MTT assay (Figure 5A). CSE-induced ALDH3A1 expression was reduced by $70 \%$ in the presence of ALDH3A1 siRNA (Figure 5B). However, either mock or scrambled siRNA alone increased ALDH3A1. 
ALDH enzymatic activity is increased by CSE (see Figure 2C); however, the question remains whether this is primarily due to induction of ALDH3A1. In HBEC2 cells, siRNA targeting ALDH3A1 prevented the CSE-induced increase in ALDH enzymatic activity (Figure 5C). Identical results were obtained when AHR expression was suppressed using siRNA. These results suggest that CSE-induced ALDH activity is likely primarily dependent on AHR-mediated ALDH3A1 induction. It is also important to note that scrambled siRNA transfection alone increased ALDH activity. When compared to the scrambled control siRNA, either AHR or ALDH3A1 siRNA significantly reduced the ALDH activity in the absence of CSE (Figure 5C).

\section{DISCUSSION}

In this study, we show that ALDH3A1 is the most robustly induced isozyme among the ALDH superfamily in CSE-exposed primary HBECs. Our studies demonstrate that CSE robustly upregulates ALDH enzymatic activity in HBECs through a mechanism dependent on AHR-mediated ALDH3A1 expression. Enforced expression of ALDH3A1 attenuates CSE-induced cytotoxicity, DNA damage and FANCD2 downregulation. By contrast, suppression of ALDH3A1 augments CSE-induced cytotoxicity and blocks the ALDH enzymatic activity. These data suggest an important role of ALDH3A1 in airway epithelial cell survival by protecting from DNA damage-induced cell death.

While other ALDH isozymes including ALDH1A3, ALDH2, ALDH3A2, ALDH3B1, ALDH5A1, ALDH19A1, ALDH16A1 and ALDH18A1 were significantly upregulated in CSE-exposed primary HBECs, none of the nineteen ALDH isozymes was downregulated by CSE. Previous human studies have demonstrated that both gene and protein expression of ALDH3A1 are upregulated in airway epithelial cells of smokers relative to those of nonsmokers [29-30]. Patel and colleagues also showed that immunohistochemical expression of ALDH3A1 protein is significantly increased in non-small cell lung cancer cells relative to normal pneumocytes or small cell lung cancer cells [29]. However, a potential role of ALDH3A1 in malignant transformation remains to be determined.

In this study, we determined the effects of CSE on ALDH enzymatic activity in HBECs. We found that CSE markedly increased ALDH activity in immortalized HBEC2 cells. CSEinduced increase in ALDH activity appeared to be attributable to ALDH3A1 given that siRNA targeting of ALDH3A1 completely blocked CSE-induced ALDH enzymatic activity. Interestingly, a previous in vitro study using human blood cells (consisting of $>99.5 \%$ red blood cells) demonstrated that CSE inhibited ALDH enzymatic activity in a dose-dependent manner [31]. Also, in the current study CSE exposure did not increase ALDH3A1 expression in primary human lung fibroblasts (data not shown). When taken together, these results would suggest that the effects of CS on ALDH activity are cell type-specific.

We further investigated the role of ALDH3A1 in modulating the cytotoxic effects of CSE by genetically-manipulating cellular levels of ALDH3A1. We showed that overexpression of ALDH3A1 attenuates CSE-induced cytotoxicity in cultured immortalized HBECs. Prior endogenous induction of ALDH3A1 by pre-exposure to a low $(0.5 \%)$ concentration of CSE also reduced subsequent CSE-induced cytotoxicity. However, it is known that CS exposure significantly alters expression of numerous genes (e.g., up-regulating multiple genes encoding antioxidant enzymes to scavenge ROS or oxidize aldehydes) in the lung of small animals [32] and in HBECs [33]. We thus speculate that the protective effects of CSEpretreatment are likely dependent on multiple pathways. However, we think that the availability of ALDH3A1 before CSE exposure is still important for cell survival against CSE because ALDH3A1 overexpression was sufficient to attenuate CSE-induced cytotoxicity. It should be also noted that the transfection procedure alone modestly increases 
ALDH3A1 expression and activity, possibly due to cellular stresses caused by transfection. The present data are consistent with previous reports demonstrating that ALDH3A1transfected cells are more resistant to cytotoxicity by a variety of genotoxins, including hydrogen peroxide, 4-HNE, mitomycin C, etoposide, and ultraviolet light [7, 9]. Notably, ALDH3A1-transfected cells have also been shown to exhibit more resistance to glutathione depletion in response to DNA-damaging agents [9]. In sum, these observations suggest that ALDH3A1 plays a crucial role in cell survival against oxidative stress induced by genotoxins.

The present studies also suggest that ALDH3A1 overexpression is sufficient to protect against CSE-induced DNA damage and FANCD2 downregulation. A previous in vitro study in immortalized HBECs demonstrated that FANCD2 overexpression protects against CSEinduced cell death and FANCD2 loss augments the effects of CSE [20]. Moreover, Hays and colleagues showed that exposure to CSE downregulates FANCD2 via translational inhibition [20]. Given that FANCD2 is a key player in the Fanconi anemia pathway involved in repair of ICLs derived from reactive aldehydes [18], the protective effects of ALDH3A1 on DNA damage and cytotoxicity may be, in part, mediated by FANCD2 expression.

Loss of AHR by siRNA transfection markedly suppressed CSE-induced ALDH3A1 upregulation in HBECs. These data suggest that ALDH3A1 upregulation by CSE is primarily mediated by AHR. Although ALDH3A1 can be upregulated through non-AHR dependent pathways, such as Pax6 and Oct1 [4-5], it is unlikely that these pathways contribute significantly to CSE-mediated induction of ALDH3A1. A recent in vitro study demonstrated that suppression of AHR levels augments CSE-induced cell death in cultured mouse lung epithelial cells [34]. Rico de Souza and colleagues attributed enhancement of CSE-induced apoptosis to down-regulation of both CuZn superoxide dismutase (CuZnSOD) and mitochondrial SOD expression in AHR-deficient cells [34]. However, the present study suggests that suppression of ALDH3A1 expression due to AHR loss may also contribute to the augmented CSE-induced cell death. ALDH3A1 catalyzes the oxidation of various LPOderived aldehydes, including 4-HNE [2]. A previous human study demonstrated that 4-HNE levels are elevated in the lungs of smokers with chronic obstructive pulmonary disease (COPD) compared with those of smokers without COPD [35], suggesting a potential role of aldehyde metabolism in the pathogenesis of COPD. Other known preferred substrates of ALDH3A1 are medium-chain length aliphatic and aromatic aldehydes [2], both of which are present in CS [36]. However, the other aldehyde components in CS, including formaldehyde, acrolein, and acetaldehydes, are not oxidized by ALDH3A1 [2]. Accordingly, the protective effects of ALDH3A1 on CSE-induced cytotoxicity are likely associated with detoxification of 4-HNE, medium-chain length aliphatic and aromatic aldehydes. Further studies will be required to determine the effects of ALDH3A1 modulation on CS-induced COPD.

\section{Acknowledgments}

This manuscript was supported in part by ALA Biomedical Research Grant: RG-231988-N, Clinical and Translational Science Center 003-2, AG037768-01, 10GRNT3530045 (to TN), HL068111 and ES015482 (to YT), the Tobacco Master Settlement through a cooperative research agreement with the University of New Mexico, DK065988 (to SHR) and EY11490 and EY17963 (VV).

\section{Abbreviation}
AHR
aryl hydrocarbon receptor
ALDH
aldehyde dehydrogenase 


\begin{tabular}{|c|c|}
\hline ALDH3A1 OE & ALDH3A1 overexpression \\
\hline ANOVA & analysis of variance \\
\hline ATM & teleangiectasia mutated protein \\
\hline CDK & cyclin dependent kinase \\
\hline COPD & chronic obstructive pulmonary disease \\
\hline $\mathbf{C S}$ & cigarette smoke \\
\hline CSE & cigarette smoke extract \\
\hline DDR & DNA damage response \\
\hline DSB & DNA double-strand break \\
\hline FA & Fanconi anemia \\
\hline FANCD2 & a member of the FA complementation group (FANC) \\
\hline 4-HNE & 4-hydroxy-2-nonenal \\
\hline HBEC & human bronchoepithelial cells \\
\hline ICLs & DNA interstrand crosslinks \\
\hline LPO & lipid peroxidation \\
\hline MTT & 3-(4,5-dimethythiazol-2-yl)-2,5-diphenyl tetrazolium bromide \\
\hline NS & not significant \\
\hline PAHs & polycyclic aromatic hydrocarbons \\
\hline PIKKs & phosphoinositide 3-kinase related protein kinases \\
\hline ROS & reactive oxygen species \\
\hline RONS & reactive oxygen/nitrogen species \\
\hline SEM & standard error of the mean \\
\hline SiRNA & small interfering RNA \\
\hline SOD & superoxide dismutase \\
\hline TB & trypan blue \\
\hline TCDD & dioxin, 2,3,7,8-tetrachlorodibenzo-p-dioxin \\
\hline
\end{tabular}

\section{References}

1. Hoffmann D, Hoffmann I. The changing cigarette, 1950-1995. J Toxicol Environ Health. 1997; 50:307-364. [PubMed: 9120872]

2. Marchitti SA, Brocker C, Stagos D, Vasiliou V. Non-P450 aldehyde oxidizing enzymes: the aldehyde dehydrogenase superfamily. Expert opinion on drug metabolism \& toxicology. 2008; 4:697-720. [PubMed: 18611112]

3. Vasiliou V, Reuter SF, Kozak CA, Nebert DW. Mouse dioxin-inducible cytosolic aldehyde dehydrogenase-3: AHD4 cDNA sequence, genetic mapping, and differences in mRNA levels. Pharmacogenetics. 1993; 3:281-290. [PubMed: 8148869]

4. Korkalainen MK, Torronen AR, Karenlampi SO. Comparison of expression of aldehyde dehydrogenase 3 and CYP1A1 in dominant and recessive aryl hydrocarbon hydroxylase-deficient mutant mouse hepatoma cells. Chem Biol Interact. 1995; 94:121-134. [PubMed: 7828219] 
5. Davis J, Davis D, Norman B, Piatigorsky J. Gene expression of the mouse corneal crystallin Aldh3a1: activation by Pax6, Oct1, and p300. Invest Ophthalmol Vis Sci. 2008; 49:1814-1826. [PubMed: 18436815]

6. Canuto RA, Muzio G, Ferro M, Maggiora M, Federa R, Bassi AM, Lindahl R, Dianzani MU. Inhibition of class-3 aldehyde dehydrogenase and cell growth by restored lipid peroxidation in hepatoma cell lines. Free Radic Biol Med. 1999; 26:333-340. [PubMed: 9895224]

7. Pappa A, Chen C, Koutalos Y, Townsend AJ, Vasiliou V. Aldh3a1 protects human corneal epithelial cells from ultraviolet- and 4-hydroxy-2-nonenal-induced oxidative damage. Free Radic Biol Med. 2003; 34:1178-1189. [PubMed: 12706498]

8. Uma L, Hariharan J, Sharma Y, Balasubramanian D. Corneal aldehyde dehydrogenase displays antioxidant properties. Exp Eye Res. 1996; 63:117-120. [PubMed: 8983957]

9. Lassen N, Pappa A, Black WJ, Jester JV, Day BJ, Min E, Vasiliou V. Antioxidant function of corneal ALDH3A1 in cultured stromal fibroblasts. Free Radic Biol Med. 2006; 41:1459-1469. [PubMed: 17023273]

10. Nyunoya T, Monick MM, Klingelhutz A, Yarovinsky TO, Cagley JR, Hunninghake GW. Cigarette smoke induces cellular senescence. Am J Respir Cell Mol Biol. 2006; 35:681-688. [PubMed: 16840774]

11. Volonte D, Kahkonen B, Shapiro S, Di Y, Galbiati F. Caveolin-1 expression is required for the development of pulmonary emphysema through activation of the ATM-p53-p21 pathway. J Biol Chem. 2009; 284:5462-5466. [PubMed: 19103597]

12. Mah LJ, El-Osta A, Karagiannis TC. gammaH2AX: a sensitive molecular marker of DNA damage and repair. Leukemia. 2010; 24:679-686. [PubMed: 20130602]

13. Sak A, Stuschke M. Use of gammaH2AX and other biomarkers of double-strand breaks during radiotherapy. Semin Radiat Oncol. 2010; 20:223-231. [PubMed: 20832014]

14. Jackson SP, Bartek J. The DNA-damage response in human biology and disease. Nature. 2009; 461:1071-1078. [PubMed: 19847258]

15. Kim H, Liu X, Kobayashi T, Conner H, Kohyama T, Wen FQ, Fang Q, Abe S, Bitterman P, Rennard SI. Reversible cigarette smoke extract-induced DNA damage in human lung fibroblasts. Am J Respir Cell Mol Biol. 2004; 31:483-490. [PubMed: 15256382]

16. Aoshiba K, Zhou F, Tsuji T, Nagai A. Dna damage as a molecular link in the pathogensis of copd in smokers. Eur Respir J. 2012

17. Stone MP, Cho YJ, Huang H, Kim HY, Kozekov ID, Kozekova A, Wang H, Minko IG, Lloyd RS, Harris TM, Rizzo CJ. Interstrand DNA cross-links induced by alpha,beta-unsaturated aldehydes derived from lipid peroxidation and environmental sources. Acc Chem Res. 2008; 41:793-804. [PubMed: 18500830]

18. Deans AJ, West SC. DNA interstrand crosslink repair and cancer. Nat Rev Cancer. 2011; 11:467480. [PubMed: 21701511]

19. Langevin F, Crossan GP, Rosado IV, Arends MJ, Patel KJ. Fancd 2 counteracts the toxic effects of naturally produced aldehydes in mice. Nature. 2011; 475:53-58. [PubMed: 21734703]

20. Hays LE, Zodrow DM, Yates JE, Deffebach ME, Jacoby DB, Olson SB, Pankow JF, Bagby GC. Cigarette smoke induces genetic instability in airway epithelial cells by suppressing FANCD2 expression. Br J Cancer. 2008; 98:1653-1661. [PubMed: 18475298]

21. Mebratu YA, Schwalm K, Smith KR, Schuyler M, Tesfaigzi Y. Cigarette smoke suppresses bik to cause epithelial cell hyperplasia and mucous cell metaplasia. Am J Respir Crit Care Med. 2011; 183:1531-1538. [PubMed: 21317312]

22. Ramirez RD, Sheridan S, Girard L, Sato M, Kim Y, Pollack J, Peyton M, Zou Y, Kurie JM, Dimaio JM, Milchgrub S, Smith AL, Souza RF, Gilbey L, Zhang X, Gandia K, Vaughan MB, Wright WE, Gazdar AF, Shay JW, Minna JD. Immortalization of human bronchial epithelial cells in the absence of viral oncoproteins. Cancer Res. 2004; 64:9027-9034. [PubMed: 15604268]

23. Wang Q, Chen W, Xu X, Li B, He W, Padilla MT, Jang JH, Nyunoya T, Amin S, Wang X, Lin Y. RIP1 potentiates BPDE-induced transformation in human bronchial epithelial cells through catalase-mediated suppression of excessive reactive oxygen species. Carcinogenesis. 2013 
24. Xu X, Bai L, Chen W, Padilla MT, Liu Y, Kim KC, Belinsky SA, Lin Y. MUC1 contributes to BPDE-induced human bronchial epithelial cell transformation through facilitating EGFR activation. PLoS ONE. 2012; 7:e33846. [PubMed: 22457794]

25. Nyunoya T, Monick MM, Powers LS, Yarovinsky TO, Hunninghake GW. Macrophages survive hyperoxia via prolonged ERK activation due to phosphatase down-regulation. J Biol Chem. 2005; 280:26295-26302. [PubMed: 15901735]

26. Nyunoya T, Monick MM, Klingelhutz AL, Glaser H, Cagley JR, Brown CO, Matsumoto E, AykinBurns N, Spitz DR, Oshima J, Hunninghake GW. Cigarette smoke induces cellular senescence via Werner's syndrome protein down-regulation. Am J Respir Crit Care Med. 2009; 179:279-287. [PubMed: 19011155]

27. Estey T, Chen Y, Carpenter JF, Vasiliou V. Structural and functional modifications of corneal crystallin ALDH3A1 by UVB light. PLoS ONE. 2010; 5:e15218. [PubMed: 21203538]

28. Murphy G Jr, Rouse RL, Polk WW, Henk WG, Barker SA, Boudreaux MJ, Floyd ZE, Penn AL. Combustion-derived hydrocarbons localize to lipid droplets in respiratory cells. Am J Respir Cell Mol Biol. 2008; 38:532-540. [PubMed: 18079490]

29. Patel M, Lu L, Zander DS, Sreerama L, Coco D, Moreb JS. ALDH1A1 and ALDH3A1 expression in lung cancers: correlation with histologic type and potential precursors. Lung cancer (Amsterdam, Netherlands). 2008; 59:340-349.

30. Tilley AE, Harvey BG, Heguy A, Hackett NR, Wang R, O'Connor TP, Crystal RG. Downregulation of the notch pathway in human airway epithelium in association with smoking and chronic obstructive pulmonary disease. Am J Respir Crit Care Med. 2009; 179:457-466. [PubMed: 19106307]

31. Helander A, Lowenmo C, Wikstrom T, Curvall M. Inhibition of human blood aldehyde dehydrogenase activity by cigarette-smoke condensate. Life Sci. 1991; 49:1901-1905. [PubMed: 1745106]

32. Rangasamy T, Misra V, Zhen L, Tankersley CG, Tuder RM, Biswal S. Cigarette smoke-induced emphysema in A/J mice is associated with pulmonary oxidative stress, apoptosis of lung cells, and global alterations in gene expression. Am J Physiol Lung Cell Mol Physiol. 2009; 296:L888-900. [PubMed: 19286929]

33. Spira A, Beane J, Shah V, Liu G, Schembri F, Yang X, Palma J, Brody JS. Effects of cigarette smoke on the human airway epithelial cell transcriptome. Proc Natl Acad Sci U S A. 2004; 101:10143-10148. [PubMed: 15210990]

34. Rico de Souza A, Zago M, Pollock SJ, Sime PJ, Phipps RP, Baglole CJ. Genetic ablation of the aryl hydrocarbon receptor causes cigarette smoke-induced mitochondrial dysfunction and apoptosis. J Biol Chem. 2011; 286:43214-43228. [PubMed: 21984831]

35. Rahman I, van Schadewijk AA, Crowther AJ, Hiemstra PS, Stolk J, MacNee W, De Boer WI. 4Hydroxy-2-nonenal, a specific lipid peroxidation product, is elevated in lungs of patients with chronic obstructive pulmonary disease. Am J Respir Crit Care Med. 2002; 166:490-495. [PubMed: 12186826]

36. Pang X, Lewis AC. Carbonyl compounds in gas and particle phases of mainstream cigarette smoke. Sci Total Environ. 2011; 409:5000-5009. [PubMed: 21925713] 
A.

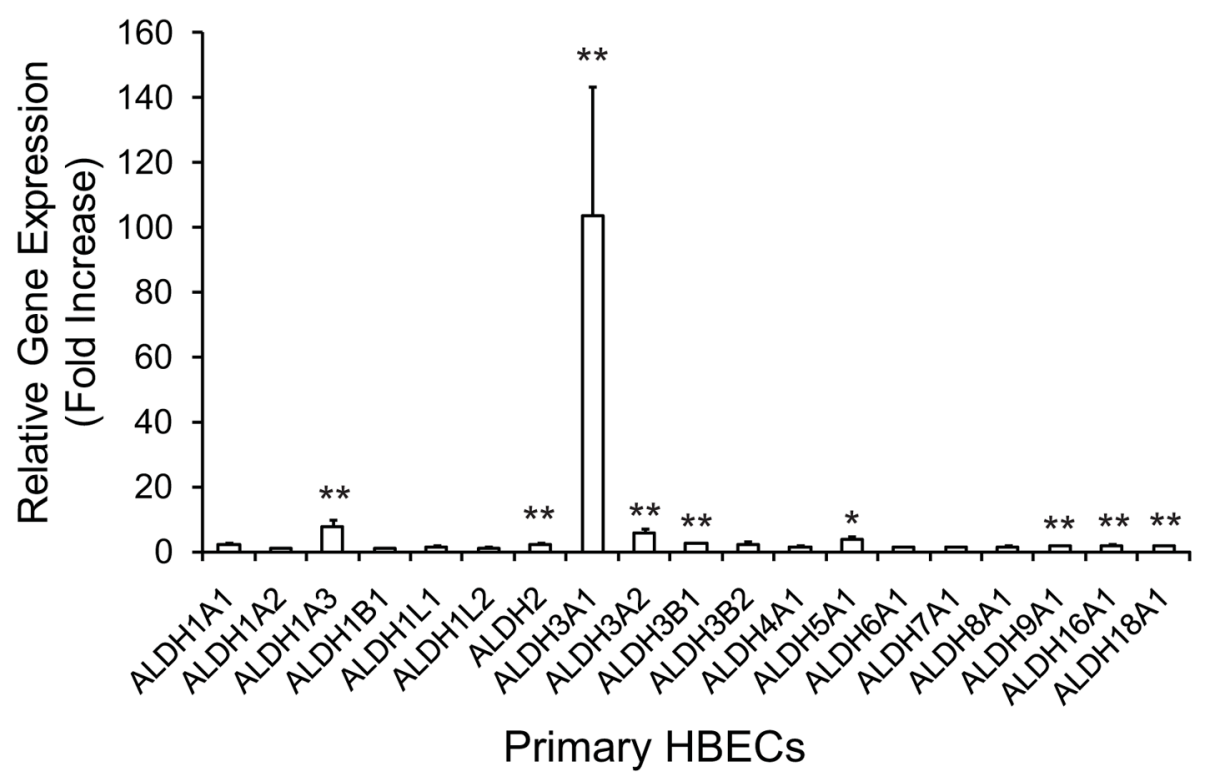

B.

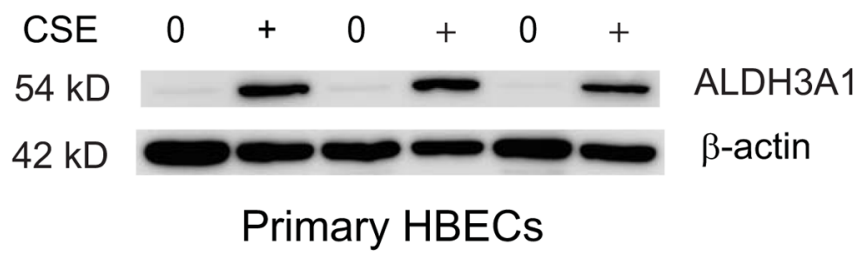

Figure 1. Primary human bronchial epithelial cells exposed to cigarette smoke extract exhibit a robust increase in ALDH3A1

Primary HBEC cells isolated from non-smoking donors were cultured in the absence or presence of $1.5 \%$ CSE for $24 \mathrm{~h}$. A. Steady-state levels of mRNA of the 19 known isozymes of ALDH were measured by RT-PCR and presented as relative fold difference compared with Actb between CSE-treated and non-treated control cells. Data are expressed as mean \pm SEM from 5 donors $(*, p<0.05 ; * *, p<0.01)$. B. Primary HBECs isolated from three nonsmoking donors were cultured in the absence $(0)$ or presence $(+)$ of $1.5 \% \mathrm{CSE}$ for $24 \mathrm{~h}$. Immunoblot analysis of ALDH3A1 was performed. 
A.

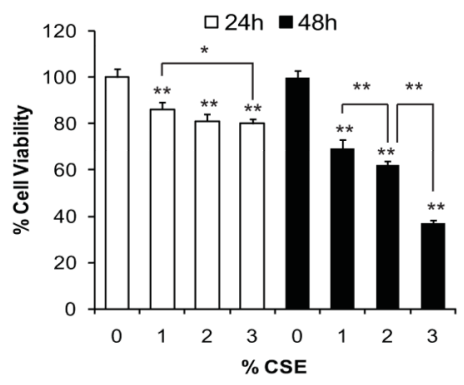

B

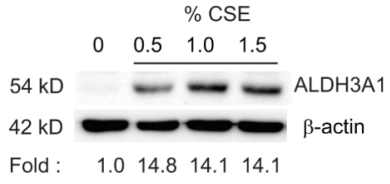

C.

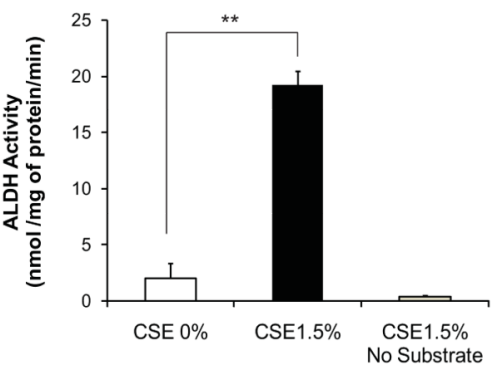

Figure 2. Cigarette smoke extract-induced ALDH3A1 protein accumulation is accompanied by increased ALDH activity

A. HBEC2 cells were cultured in the presence or absence of CSE $(0,1,2$, and $3 \%)$ for 24 or 48h. Cell viability was determined by the MTT assay. Data are expressed as mean \pm SEM for two independent experiments with three triplicate samples $(*, p<0.05 ; * *, p<0.01)$. B. HBEC2 cells were cultured in the absence $(0)$ or presence of CSE $(0.5,1.0$, and $1.5 \%)$. Immunoblot analysis of ALDH3A1 was performed $24 \mathrm{~h}$ thereafter. Data are representative of three experiments. C. At the end of a $24 \mathrm{~h}$ period of treatment without (CSE 0\%) or with $1.5 \%$ CSE (CSE 1.5\%), ALDH enzymatic activity was measured in HBEC2 cells using benzaldehyde (as a substrate) to initiate the reaction. Enzyme activity was also measured in CSE-treated cells to which no benzaldehyde (CSE 1.5\% No substrate) was added to determine background $\mathrm{NAD}(\mathrm{P}) \mathrm{H}$ production. Data are expressed as mean $\pm \mathrm{SEM}$ from three independent experiments with three triplicate samples $(* *, p<0.01)$. 
A.

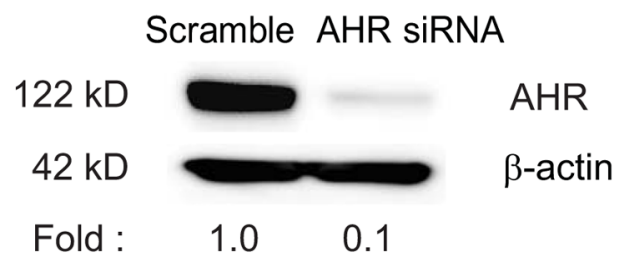

B.

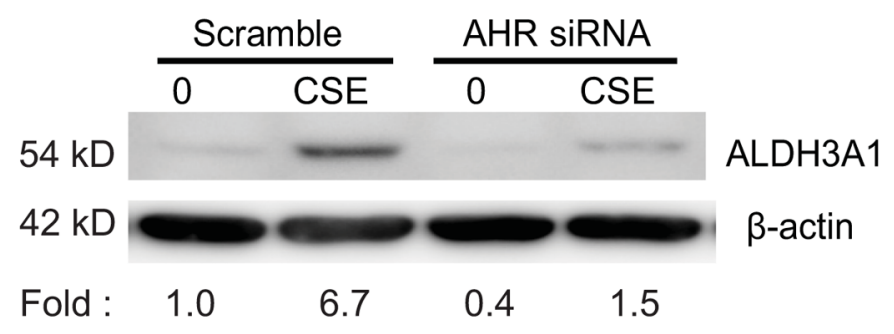

Figure 3. Cigarette smoke extract induces ALDH3A1 via aryl hydrocarbon receptor A. HBEC2 cells were transfected with either siRNA targeting AHR (AHR siRNA) or the scrambled siRNA (Scrambled) as control. Immunoblot analysis of AHR was performed $24 \mathrm{~h}$ after transfection. B. HBEC2 cells were treated as in A. and then incubated in the absence (0) or presence of $1.5 \%$ CSE (CSE). Immunoblot analysis of ALDH3A1 was performed 24 $\mathrm{h}$ thereafter. Data are representative of three experiments. 
A.

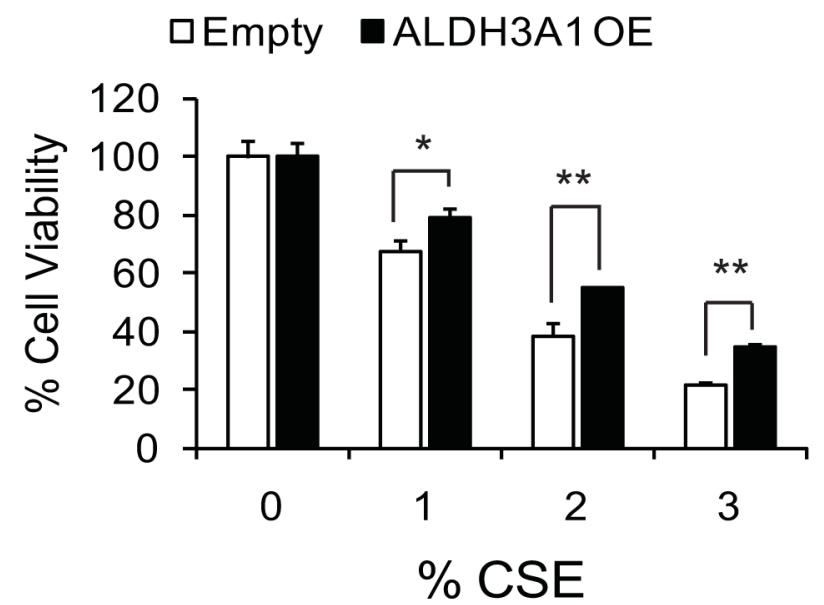

B.

$\frac{\text { Empty }}{0 \quad \mathrm{CSE}} \frac{\text { ALDH3A1 OE }}{0 \quad \mathrm{CSE}}$

$54 \mathrm{kD}$

ALDH3A1

$16 \mathrm{kD}$

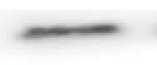

$\longrightarrow$

phosH2AX

$42 \mathrm{kD}$

$\beta$-actin

C.

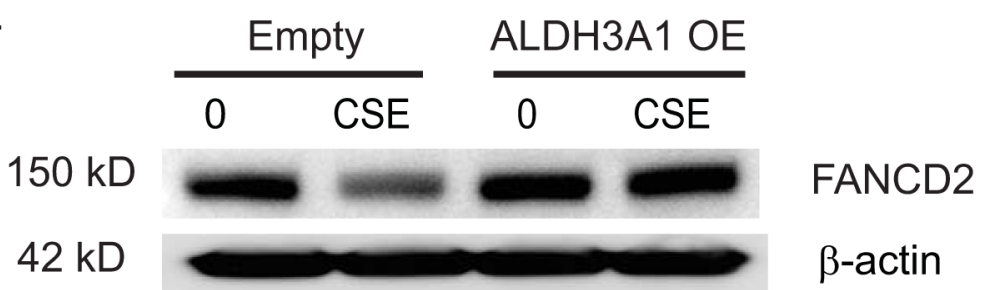


D.

\section{$\square$ No Prior CSE םPrior CSE}

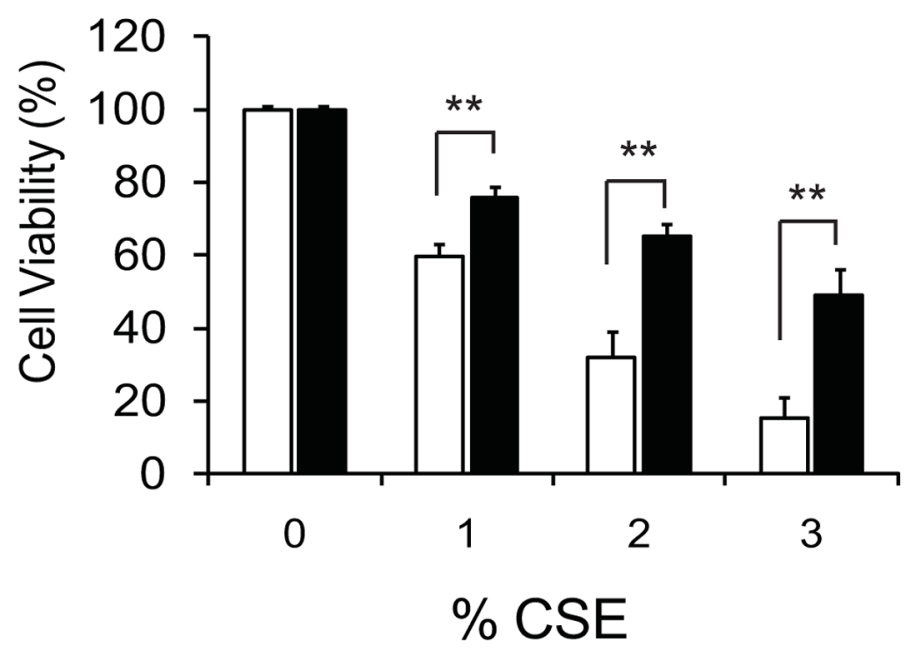

Figure 4. ALDH3A1 attenuates cigarette smoke extract-induced cytotoxicity, DNA damage, and FANCD2 downregulation

HBEC2 cells were transduced with a lentiviral vector (pReceiver) encoding ALDH3A1 cDNA or the empty vector and selected with $25 \mu \mathrm{g} / \mathrm{mL}$ hygromycin. HBEC 2 cells treated with empty vector (Empty) or overexpressing ALDH3A1 (ALDH3A1 OE) were cultured in the absence (0\%) or presence of CSE $(1,2$, and $3 \%)$ for $48 \mathrm{~h}$. A. Cell viability was determined using the MTT assay at $48 \mathrm{~h}$. Data are expressed as mean \pm SEM for three independent experiments (*, $p<0.05 ; * *, p<0.01)$. B. Immunoblot analysis of ALDH3A1 and phosphorylated $\mathrm{H} 2 \mathrm{AX}$ (phos $\mathrm{H} 2 \mathrm{AX}$ ) was performed $24 \mathrm{~h}$ after treatment without (0) or with $1.5 \%$ CSE (CSE). Immunoblotting data are representative of three experiments. C. HBEC2 cells were treated as in B. Immunoblot analysis of FANCD2 was performed. Data are representative of three experiments. D. HBEC2 cells were pretreated with vehicle (No Prior CSE) or $0.5 \%$ CSE (Prior CSE) for $24 \mathrm{~h}$, and then further incubated in the absence (0) or presence of CSE $(1,2$, and $3 \%)$ for additional $48 \mathrm{~h}$. Cell viability was determined using the MTT assay at $48 \mathrm{~h}$. Cell viability was expressed as a percentage of the absorbance intensity relative to the control cells without (No Prior CSE) or with CSE-pretreatment (Prior CSE) $48 \mathrm{~h}$ after culture in the absence of CSE. Data are expressed as mean \pm SEM for three independent experiments $(* *, p<0.01)$. 


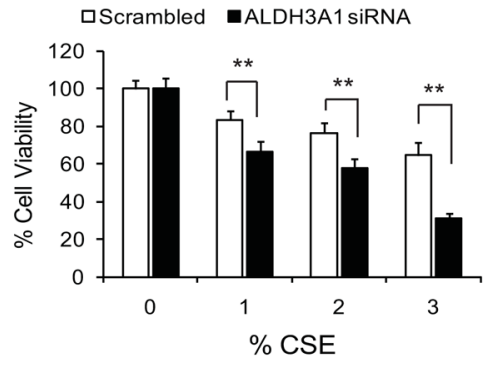

MTT Assay

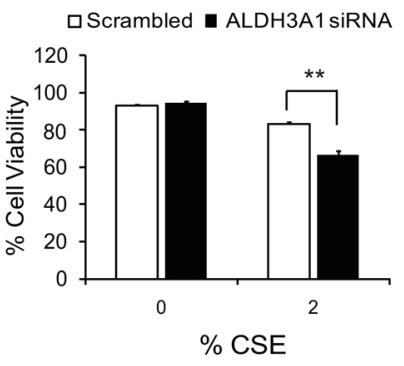

Trypan Blue Assay

B.

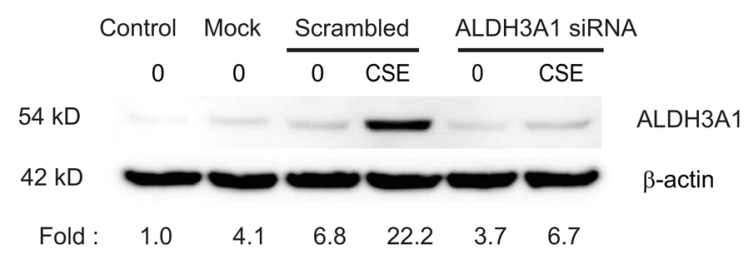

C.

口 CSE $0 \%$ CSE $1.5 \%$

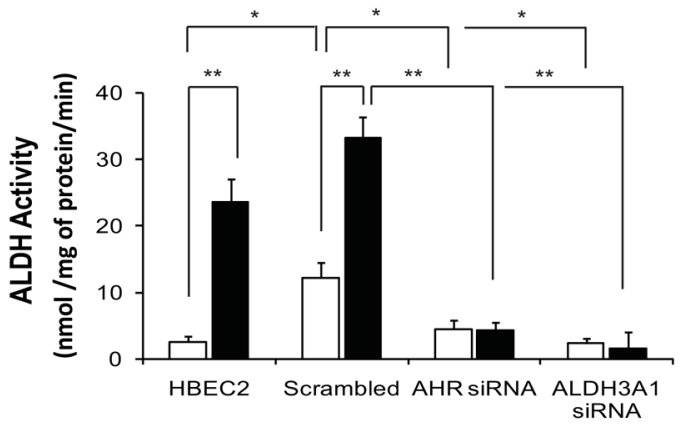

Figure 5. Suppression of ALDH3A1 levels both blocks ALDH enzymatic activity and augments cytotoxicity induced by cigarette smoke extract

A. HBEC2 cells transfected with siRNA targeting ALDH3A1 mRNA (ALDH3A1 siRNA) or the scrambled control (Scrambled) were cultured for $24 \mathrm{~h}$ followed by further incubation in the absence $(0 \%)$ or presence of CSE $(1,2$, or $3 \%)$. Cell viability was determined using the MTT assay and trypan blue assay (only $2 \% \mathrm{CSE}$ ) at $48 \mathrm{~h}$. Data are expressed as mean \pm SEM from two independent experiments with triplicate samples $(* *, p<0.01)$. B. HBEC2 cells were treated as in A, except for including the control (no treatment) and mock (reagent only). Immunoblot analysis of ALDH3A1 was performed $24 \mathrm{~h}$ after treatment without (0) or with $1.5 \%$ CSE (CSE). Data are representative of three experiments. C. HBEC2 cells were treated as in A. In addition, some HBEC2 cells were not transfected (Control) or transfected with siRNA targeting AHR mRNA (AHR siRNA), ALDH3A1 mRNA (ALDH3A1 siRNA), or the scrambled siRNA (Scrambled). ALDH enzymatic activity was measured spectrophotometrically through the measurement of $\mathrm{NAD}(\mathrm{P}) \mathrm{H}$, a byproduct of benzaldehyde (substrate) oxidation by ALDH. Data are expressed as mean \pm SEM from three independent experiments with three triplicate samples $(*, p<0.05 ; * *, p<0.01)$. 